\section{Serumtryptase als Risikofaktor bestätigt}

\author{
Allergische Reaktionen auf Bienen- oder Wespenstiche sind vielfach \\ systemisch, in bis zu einem Fünftel dieser Fälle sogar lebens- \\ bedrohlich. Welche Faktoren prädisponieren für einen schweren \\ Verlauf und wie lassen sie sich prognostisch nutzen?
}

\begin{abstract}
$\mathrm{M}$ öglichen Korrelationen zwischen schweren Anaphylaxien nach $\mathrm{Hy}$ menopterenstichen einerseits und Serumtryptasewerten, Alter, Geschlecht, kardiovaskulärer Medikation sowie vorangehenden schwächeren systemischen Reaktionen der Gestochenen andererseits auf die Spur zu kommen, war das Ziel einer multizentrischen europäischen Studie. Ein Allergologenteam wertete dazu retrospektiv Daten von 962 Patienten aus, die nach einem Bienen- oder Wespenstich unter Feldbedingungen eine systemische Reaktion entwickelt hatten. Als schwere Reaktionen galten anaphylaktischer Schock, Bewusstlosigkeit oder Herz- und Atemstillstand.

206 Patienten $(21,4 \%)$ hatten auf den Stich hin eine solche schwere Reaktion entwickelt. Männern waren davon häufiger betroffen als Frauen, und die Wahrscheinlichkeit eines adversen Verlaufs nahm mit steigendem Alter zu. Als
\end{abstract}

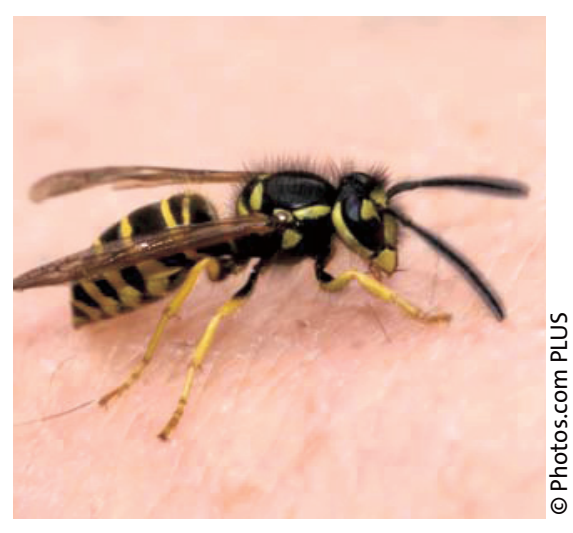

Das Risiko, nach einem Wespenstich eine schwere systemische Reaktion zu erleiden, korreliert direkt mit der Serumtryptasekonzentration.

pharmakologischer Risikofaktor erwies sich die Einnahme von ACE-Hemmern zur Blutdrucksenkung. Außerdem waren schwere Reaktionen bei denjenigen häufiger, die zuvor bereits auf einen
Stich hin eine schwächere systemische Reaktion entwickelt hatten. Als Schlüsselfaktor für eine schwere systemische Reaktion konnten die Autoren aber eine erhöhte Serumtryptasekonzentration identifizieren: Bereits $\mathrm{ab}$ einem Wert von etwa $5 \mu \mathrm{g} / \mathrm{l}$ (Median der betrachteten Kohorte 4,25 $\mu \mathrm{g} / \mathrm{l}$ ) stieg das Risiko an, bei $20 \mu \mathrm{g} / \mathrm{l}$ lag es beim 3,8-Fachen. Daher empfehlen die Autoren die Bestimmung der Serumtryptase - die bei Stimulation von den Mastzellen ausgeschüttet wird - zur Risikoabschätzung.

Fazit: Diese große multinationale Studie unterstreicht die Bedeutung der Serumtryptase als Prädiktor für schwere anaphylaktische Reaktionen bei einer Bienen- und Wespengiftallergie. Außerdem ist bei Einnahme von ACE-Hemmern ist die Umstellung auf andere Blutdrucksenker empfehlenswert.

\section{$f k$}

Ruëff F et al. Predictors of severe systemic anaphylactic reactions in patients with Hymenoptera venom allergy: importance of baseline serum tryptase - a study of the European Academy of Allergology and Clinical Immunology Interest Group on Insect Venom Hypersensitivity. J Allergy Clin Immunol 2009; 124: 1047-54

\title{
Ist einmal Adrenalin zu wenig?
}

\section{Kinder mit dem Risiko einer Nahrungsmittelanaphylaxie sollten in jedem Fall mit einem Adrenalininjektor ausgestattet sein. US-ameri- kanische Allergologen wollten wissen, ob tatsächlich in allen Fällen eine Adrenalingabe ausreicht.}

$\int \mathrm{ie}$ ie Daten von 1.255 Kindern, die im Zeitraum zwischen 2001 und 2006 wegen einer nahrungsmittelinduzierten allergischen Reaktion die Notaufnahmen zweier großer Kliniken in Boston aufgesucht hatten, wurden retrospektiv ausgewertet. Es konnten 605 Fälle identifiziert werden, welche die Kriterien einer Anaphylaxie erfüllten. Das mediane Alter dieser Kinder lag bei 5,8 Jahren, 62\% waren Jungen. Als häufigste Auslöser der Reaktionen wurden Erdnüsse (23\%), Nüsse
(18\%) und Milchprodukte (15\%) nachgewiesen.

Während des Aufenthalts in der Notaufnahme erhielten von den Kindern mit Anaphylaxie 59\% Antihistaminika, 56\% Glukokortikoide, 23\% intravenöse Flüssigkeitszufuhr und 13\% inhalative $\beta$-Agonisten. In $20 \%$ der Fälle wurde Adrenalin verabreicht - meist subkutan. Über den gesamten Zeitverlauf der allergischen Reaktion - einschließlich der vorstationären Phase - erhielten 44\% der Kinder eine Adrenalindosis und $6 \%$ mindestens zwei Gaben. Von den Kindern, die Adrenalin verabreicht bekamen, benötigten also $12 \%$ mehr als eine Dosis. Als Prädiktoren für die Gabe von mehr als einer Adrenalindosis ergaben sich ein Alter von über zehn Jahren sowie die Vorbehandlung der allergischen Reaktion in einem regionalen Krankenhaus. Patienten mit Autoinjektor erhielten nicht signifikant häufiger mehrere Adrenalindosen als andere.

Fazit: Bei Kindern mit dem Risiko einer Nahrungsmittelanaphylaxie reicht eine Adrenalindosis häufig nicht aus. Daher empfiehlt sich unter Umständen das Mitführen von zwei Autoinjektoren. af

Rudders SA et al. Multicenter study of repeat epinephrine treatments for foodrelated anaphylaxis. Pediatrics 2010; 125: e711-8 